\title{
Generation of regular optical pulses in VCSELs below the static threshold
}

\author{
A.A. Krents ${ }^{1,2}$, N.E. Molevich ${ }^{1,2}$, D.A. Anchikov ${ }^{1}$, S.V. Krestin ${ }^{2}$ \\ ${ }^{I}$ Samara National Research University, Moskovskoye Shosse 34, 443086, Samara, Russia \\ ${ }^{2}$ Lebedev Physical Institute, Novo-Sadovaya Str. 221, 443011, Samara, Russia
}

\begin{abstract}
We study numerically the dynamics of a vertical-cavity surface emitting laser (VCSEL) with external optical injection and asymmetrical triangular current modulation. Even if the average current is below the threshold, the VCSEL without optical injection emits irregular optical pulses. External optical injection stabilizes the laser output, reduces the standard deviation of the generated pulses and increases their averaged amplitude. The results of this study make it possible to reduce the threshold current.
\end{abstract}

Keywords: vertical-cavity surface-emitting lasers (VCSELs); optical injection; polarization; semiconductor lasers

\section{Introduction}

Vertical cavity surface emitting lasers (VCSELs) are among the most attractive light sources in modern optical devices, especially for both digital and analog photonic communication systems. They have the low threshold current, high modulation bandwidth, and emit a single-longitudinal mode and circular output beams that result in high coupling efficiencies into optical fibers. The output radiation of VCSELs is polarized along one of the two linearly polarized modes, aligned to the crystallographic directions. Under current modulation, nonlinear effects such as period doubling, chaos and multistability can arise $[1,2]$. In this paper, using the standard spin-flip model extended to optical injection, we demonstrate that VCSEL, with asymmetric triangular current modulation and a small coherent optical injection, can generate regular optical pulses in both orthogonal polarizations.

Using asymmetric modulation makes it possible to reduce the threshold current and thermal heating of laser active medium.

\section{Model}

Polarization properties of VCSEL are described by the spin-flip model extended to optical injection [3]:

$$
\begin{aligned}
& E_{x}=k(1+i \alpha)\left[(N-1) E_{x}+i n E_{y}\right]-i\left(\gamma_{p}+\Delta \omega\right) E_{x}-\gamma_{a} E_{x}+k E_{i n j} \cos (\psi)+\sqrt{\beta_{s p}} \xi_{x}, \\
& E_{y}=k(1+i \alpha)\left[(N-1) E_{y}-i n E_{x}\right]+i\left(\gamma_{p}-\Delta \omega\right) E_{y}+\gamma_{a} E_{y}+k E_{i n j} \sin (\psi)+\sqrt{\beta_{s p}} \xi_{y}, \\
& N=\gamma_{N}\left[\mu(t)-N\left(1+\left|E_{x}\right|^{2}+\left|E_{y}\right|^{2}\right)-i n\left(E_{y} E_{x}^{*}-E_{x} E_{y}^{*}\right)\right], \\
& n=-\gamma_{s} n-\gamma_{N}\left[n\left(\left|E_{x}\right|^{2}+\left|E_{y}\right|^{2}\right)+i N\left(E_{y} E_{x}^{*}-E_{x} E_{y}^{*}\right)\right],
\end{aligned}
$$

where $k$ is the field decay rate, $\gamma_{N}$ is the decay rate of the total carrier population, $\gamma_{s}$ is the spin-flip rate which accounts for the mixing of carrier populations with different spins, $\alpha$ is the linewidth enhancement factor, $\gamma_{a}$ and $\gamma_{p}$ are linear anisotropies representing dichroism and birefringence, $\Delta \omega$ is the detuning parameter, $\Psi$ is the angle between the $\mathrm{x}$ axis? and the direction of the linearly polarized optical injection, $\beta_{s p}$ is the noise strength, $\xi_{x, y}$ are uncorrelated Gaussian white noises, and $\mu(t)$ is the normalized injection current parameter (the static threshold is-at $\mu_{t h, s}=1$ ).

The current is modulated with an asymmetric triangular periodic signal of amplitude $\Delta \mu$, rising from $\mu_{0}$ during the time interval $T_{1}$ and falling back to $\mu_{0}$ during the time interval $T_{2}$. One modulation cycle is:

$\mu(t)=\mu_{0}+\Delta \mu\left(t / T_{1}\right)$ for $0 \leq t \leq T_{1}$,

$\mu(t)=\mu_{0}+\Delta \mu\left[1-\left(t-T_{1}\right) / T_{2}\right]$ for $T_{1} \leq t \leq T_{1}+T_{2}$.

The average current, $\mu_{\text {ave }}=\mu_{0}+\Delta \mu / 2$, is independent of the modulation period, $T=T_{1}+T_{2}$. The asymmetry of the modulation is characterized by the parameter $\alpha_{a}=T_{1} / T$ with $0 \leq \alpha_{a} \leq 1$.

\section{Results and Discussion}

The equations were simulated with typical VCSEL parameters [4]: $k=300 n s^{-1}, \alpha=3, \gamma_{N}=1 n s^{-1}, \gamma_{a}=0.5 n s^{-1}$, $\gamma_{p}=50 \mathrm{rad} / \mathrm{s}, \gamma_{s}=50 \mathrm{~ns}^{-1}$, and $\beta_{s p}=10^{-6} \mathrm{~ns}^{-1}$. Asymmetrical triangular modulation of current leads to the generation of irregular optical pulses even if, on average, the current is below the threshold [3]. There is an optimal modulation asymmetry, typically $\alpha_{a} \cong 0.8$, for which the averaged intensity and the averaged pulse amplitude reach their maximum value, and for this asymmetry, the dispersion of the pulse amplitude reaches its minimum value. Figure 1(a)-(f) displays time traces of the 
$I_{x}=\left|E_{x}\right|^{2}$ and $I_{y}=\left|E_{y}\right|^{2}$ fomputer Optics and Nanophotonics / A.A. Krents, N.E. Molevich, D.A. Anchikov, S.V. Krestin $\mu_{\text {ave }}=0.87$, asymmetry $\alpha_{a}=0.8$, frequency detuning between injection and laser mode $\Delta \omega=0, \Psi=\pi / 4$ (injection in both polarizations is equal).
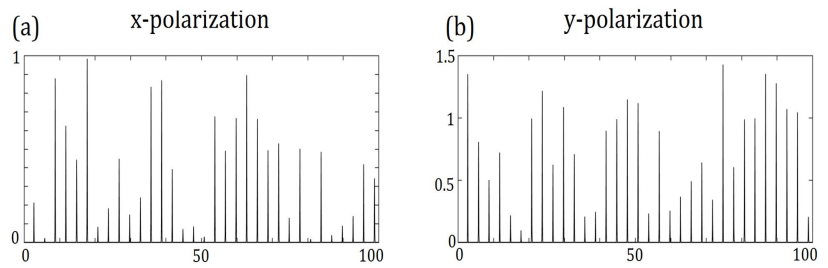

(c)

(d)
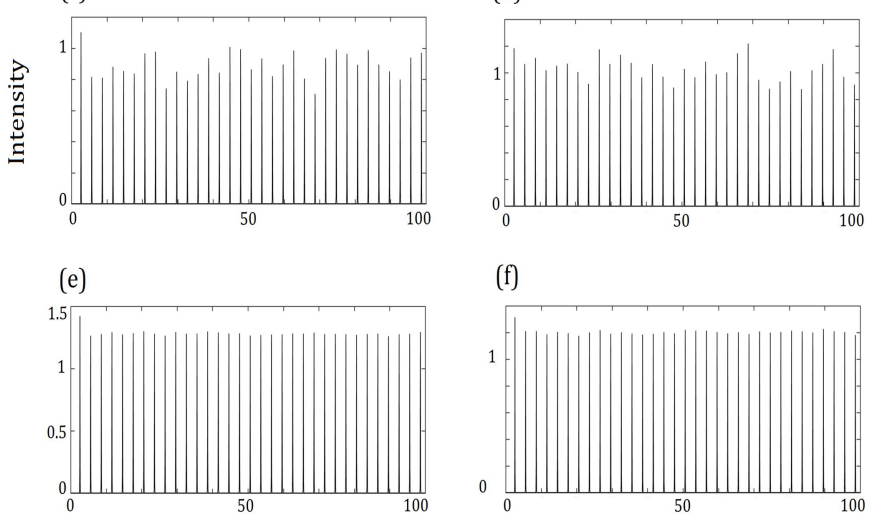

(f)

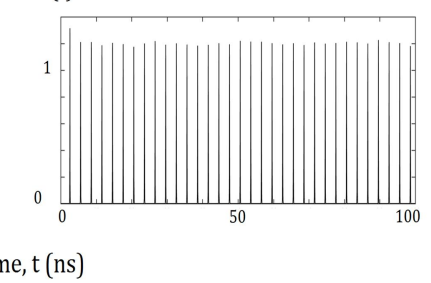

Fig.1. Time traces of intensities of the orthogonal linear polarization: (a), (b) $E_{i n j}=0$, (c), (d) $E_{i n j}=10^{-5}$, (e), (f) $E_{i n j}=10^{-4}$.

Figure 2(a) displays standard deviation of the intensity of the pulse, depending on the value of the optical injection. It can be observed that injection of the optical signal leads to more regular pulses. Also, injection leads to increasing of the mean value of generated pulse amplitude for both polarizations (Figure 2(b)). Thus, optical injection stabilizes the laser output and increases the laser efficiency.
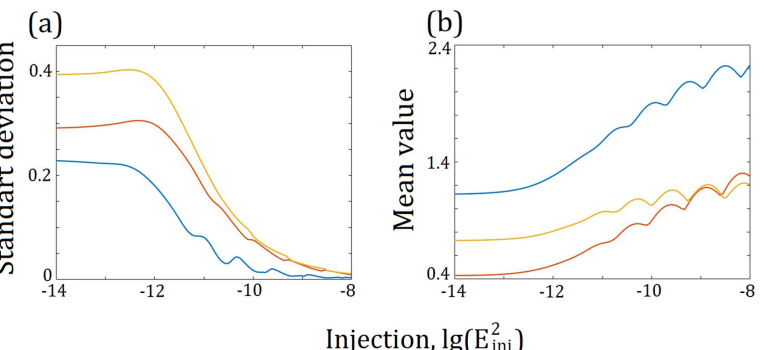

Fig.2. Standard deviation of the intensity of the pulses (a). Mean of the intensity of the pulses (b). Red is the x-polarization, yellow is the y-polarization and blue is the total intensity.

Figure 3(a) displays the standard deviation of the total intensity of the pulses, depending on the value of the injection angle. Figure 3(b) displays the mean of the total intensity of the pulses. The mean of the total intensity has the maximum value for the angle $\Psi=\pi / 2$ (parallel optical injection). Standard deviation has minimum for $\Psi=\pi / 2$ and $\Psi=0$. X-polarization vanishes for parallel optical injection and y-polarization vanishes for orthogonal optical injection $(\Psi=0)$. Thus, the optical injection in the $\mathrm{y}$-mode is the most benefit.
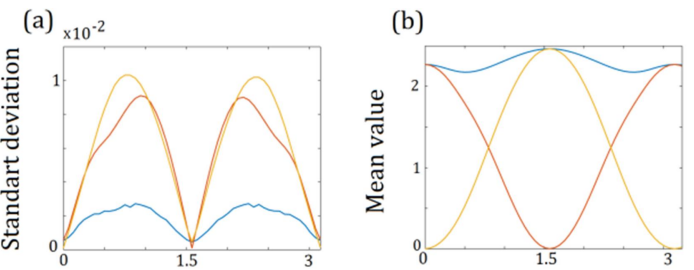

Injection angle, $\psi(\mathrm{rad})$

Fig. 3. Standard deviation of the intensity of the pulses (a). Mean of the intensity of the pulses (b). $E_{i n j}=10^{-4}$. Red is the x-polarization, yellow is the ypolarization and blue is the total intensity. 
Computer Optics and Nanophotonics / A.A. Krents, N.E. Molevich, D.A. Anchikov, S.V. Krestin

The frequency detuning between injected and generated radiation $\Delta \omega$ is the important parameter of the model. Figures 4(a), (b) display the standard deviation of the total intensity of the pulses and the mean of the total intensity of the pulses for $\Psi=\pi / 4$. The standard deviation of the total intensity of the pulses has minimum for $\Delta \omega \approx \gamma_{p}=50$ (in this case injection is coherent to the y-mode). The mean of the total intensity of the pulses has maximum for the same detuning value. For $\Psi=0$, the standard deviation of the total intensity of the pulses has minimum for $\Delta \omega=0$ and the mean of the total intensity of the pulses has maximum for the same detuning value. For $\Psi=\pi / 2$, the standard deviation of the total intensity of the pulses has minimum for $\Delta \omega \approx \gamma_{p}=50$ and the mean of the total intensity of the pulses has maximum for the same detuning value.

(a)

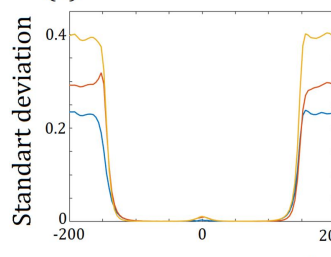

(b)

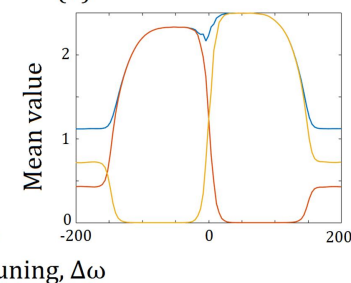

Fig.4. Standard deviation of the intensity of the pulses (a). Mean of the intensity of the pulses (b). $E_{i n j}=10^{-4}, \Psi=\pi / 4$. Red is the x-polarization, yellow is the y-polarization and blue is the total intensity.

Thus, both amplitude and polarization of pulses generated by asymmetrically modulated VCSELs can be stabilized by weak external optical injection.

\section{Conclusion}

In summary, asymmetrically periodically modulated VCSELs with a pumping current below the threshold and an external optical injection, have been numerically investigated. We have shown that injection of weak external optical signal stabilizes the VCSEL generation. We showed that generation of quasiregular optical pulses is already possible for the injection value $E_{i n j}=10^{-4}$, which is only about $5 \cdot 10^{-9}$ of the output intensity of generation. Optical injection increases the mean amplitude of generated pulses and decreases the standard deviation of the intensity of the pulses. Control of the output radiation polarization is also possible. We showed the possibility of smooth adjustment of the polarization of the generated pulses by varying the optical injection angle $\Psi$. We found that the standard deviation of the intensity of the pulses has minimum for injection in ymode (parallel optical injection). Method proposed in this paper provides a generation of regular optical pulses in VCSELs below the static threshold.

\section{Acknowledgements}

This research was supported by Russian Foundation for Basic Research (16-32-60151 mol_a_dk); State assignment to educational and research institutions under project 3.1158.2017.

\section{References}

[1] Agrawal GP. Effect of gain nonlinearities on period doubling and chaos in directly modualted semiconductor lasers. Appl. Phys. Lett. 1986; 49: 1012-1015

[2] Zamora-Munt J, Masoller C. Generation of optical pulses in VCSELs below the static threshold using asymmetric current modulation. Opt. Express 2008; 16(22): 17848-17853.

[3] San Miguel M, Feng Q, Moloney JV. Light-polarization dynamics in surface-emitting semiconductor. Phys. Rev. A 1995; 52: $1728-1739$.

[4] Martin-Regalado J, Prati F, San Miguel M, Abraham NB. Polarization properties of vertical-cavity surface- emitting lasers. IEEE J. Quantum Electron 1997; 33: $765-783$. 